\title{
Vascularização do Tendão Tibial Posterior: revisão integrativa da literatura
}

\author{
Vascularization of Posterior Tibial Tendon: integrative literature review \\ Vascularización del Tendón Tibial Posterior: revisión integradora de la literatura \\ Thiago Batista Faleiro ${ }^{1}$, Denisval Flores Cavalcanti²*.
}

\section{RESUMO}

Objetivo: Indicar os aspectos de interesse dos ortopedistas sobre as características anatômicas, aspecto histológico, sua fisiopatologia e manejo terapêutico para a degeneração do Tendão Tibial Posterior, ressaltando o fator vascularização. Métodos: Realizou-se uma revisão integrativa, mediante pesquisa nas bases de dados: Portal acadêmico do CAPES, Scielo, Biblioteca Virtual em Saúde (BVS) e PUBMED, em que foram incluídos artigos científicos e dissertações em português e inglês, no período de 2000 a 2020 . Resultados: Foram encontrados 552 artigos, sendo que, após análise dos critérios de elegibilidade, foram selecionados 21 artigos. A degeneração do TTP é uma condição comum, mas muitas vezes mal diagnosticada. Causa uma deformidade progressiva e dolorosa noemada como "pé chato". A anatomia e vascularização expõem a região do TTP a distúrbios degenerativos importantes ao ponto até de uma ruptura. Relata-se na literatura uma zona de suprimento sanguíneo baixa nesse tendão, que parece estar diretamente associado ao local de maior ferequência de lesões degenerativas. Considerações finais: Os dados da literatura mostraram uma zona de suprimento sanguíneo baixa do tendão de Gliding, que parece estar diretamente associado ao local de maior prevalência das lesões degenerativas. Entretanto, os mecanismos vasculares dessa degeneração ainda são desconhecidos e se faz necesário estudos mais aprofundados.

Palavras-chave: Tendão, Tíbia, Vasculatura.

\begin{abstract}
Objective: Indicate aspects of interest to orthopedists on anatomical characteristics, histological aspects, their pathophysiology and therapeutic management for the posterior tibial tendon (PTT) degeneration, emphasizing the vascularization factor. Methods: An integrative review was carried out by searching the databases: CAPES academic portal, Scielo, Virtual Health Library (VHL) and PUBMED, which included scientific articles and dissertations in Portuguese and English, in period 2000 to 2020. Results: 552 articles were found, and, after analyzing the eligibility criteria, 21 articles were selected. Degeneration of PTT is a common but often misdiagnosed condition. It causes a progressive and painful deformity called "flat foot". Anatomy and vascularization expose the PTT region to major degenerative disorders to the point of even a rupture. A low blood supply zone in this tendon has been reported in the literature, which appears to be directly associated with the site of greater frequency of degenerative lesions. Final considerations: Literature data showed a low blood supply zone of the Gliding tendon, which appears to be directly associated with the place of greatest prevalence of degenerative injuries. However, the vascular mechanisms of this degeneration are still unknown and further studies are needed.
\end{abstract}

Key words: Tendons, Tibia, Blood supply.

${ }^{1}$ Universidade Estadual Paulista (UNESP), Salvador - BA.

${ }^{2}$ Hospital Santo Antônio, Salvador - BA. *E-mail: cavalcanti-cavalcanti@hotmail.com 


\section{RESUMEN}

Objetivo: Indicar los aspectos de interés para los ortopedistas en cuanto a características anatómicas, aspectos histológicos, su fisiopatología y manejo terapéutico para la degeneración del tendón tibial posterior, destacando el factor de vascularización. Métodos: Se realizó una revisión integradora buscando en las bases de datos: portal académico CAPES, Scielo, Biblioteca Virtual en Salud (BVS) y PUBMED, en el que se incluyeron artículos científicos y disertaciones en portugués e inglés, en el período 2000 hasta 2020. Resultados: Se encontraron 552 artículos y, luego de analizar los criterios de elegibilidad, se seleccionaron 21 artículos. La degeneración de TTP es una condición común pero a menudo mal diagnosticada. Causa una deformidad progresiva y dolorosa llamada "pie plano". La anatomía y la vascularización exponen la región TTP a trastornos degenerativos importantes hasta el punto de incluso una ruptura. Una zona de bajo suministro de sangre en este tendón se informa en la literatura, que parece estar directamente asociada con el sitio de mayor frecuencia de lesiones degenerativas. Consideraciones finales: Los datos de la literatura muestran una zona de bajo riego sanguíneo del tendón deslizante, que parece estar directamente asociada con el lugar de mayor prevalencia de lesiones degenerativas. Sin embargo, los mecanismos vasculares de esta degeneración aún se desconocen y se necesitan más estudios.

Palabras-clave: Tendón, Tibia, Vasculatura.

\section{INTRODUÇÃO}

A função dos músculos e tendões concebe-se como fator fundamental no equilíbrio dinâmico do corpo humano. Durante a posição ortostática o arco mantém-se passivamente pela arquitetura óssea e ligamentos (PRADO MP, 2000). Entra nesse quesito, o músculo tibial posterior, ele participa do equilíbrio por sua ação sinérgica aos músculos fibular longo e curto, tríceps sural e, assim como, ao músculo tibial anterior. Essa rede muscular compõe os estabilizadores dinâmicos do tornozelo (SILVA DJF, 2012).

O músculo tibial posterior, o inversor mais potente do pé, age, principalmente, nas articulações talocalcaneonavicular e calcaneocubóide, tendo como efeito posicionar o retropé em varo e equino ao realizar a marcha (PRADO MP, 2000; GEREMIA JM, 2011).

Com a inversão do retropé e o bloqueio das principais articulações supracitadas, facilita-se a ação integrada do tríceps sural e fáscia plantar no desprendimento e sustentação do arco longitudinal medial (PRADO MP, 2000; SILVA DJF, 2012; GEREMIA JM, 2011).

A disfunção do músculo tibial posterior ocasiona alterações dinâmico-estruturais na região do pé (PRADO $M P, 2000)$. As características histológicas indicam que roturas parciais comprometem sua função e que a perda de ação resulta em alterações no comprimento do músculo (PRADO MP, 2000; SANHUDO JAV, 2012). Desse modo, associa-se o pé plano adquirido à incapacidade funcional observada pela degeneração do tendão tibial posterior (PRADO MP, 2000; SILVA DJF, 2012).

O distúrbio do tendão tibial posterior engloba um grande espectro de anomalias progressivas, tendo como encadeamento inicial a origem de uma tenossinovite até evolução para uma ruptura tendinosa, com ou sem colapso do retropé (PRADO MP, 2000; GEREMIA JM, 2011).

Dessa forma, considerando o seu impacto na qualidade de vida dos pacientes, este artigo objetiva revisar a literatura indicando os aspectos de interesse dos ortopedistas sobre as características anatômicas, aspecto histológico, sua fisiopatologia e manejo terapêutico para a degeneração do Tendão Tibial Posterior, ressaltando a fator da vascularização desse tendão nesta condição.

\section{MÉTODOS}

Trata-se de uma revisão integrativa da literatura, no qual utilizou mecanismos de busca e de estudo bibliométrico, em que foram utilizados artigos científicos e dissertações indexadas do Portal Acadêmico da 
Coordenação de Aperfeiçoamento de Pessoal de Nível Superior (CAPES); da Biblioteca Virtual de saúde (BVS); da Scientific Electronic Library Online (Scielo) e do Medical Literature Analysis and Retrieval System Online (MEDLINE/PubMed).

\section{Critérios de elegibilidade dos estudos}

Nesse estudo empregaram-se tais critérios de inclusão: artigos científicos e dissertações, publicados na íntegra, nos idiomas Português e Inglês, entre os anos de 2000 até maio de 2020, na modalidade de revisão de literatura, revisão sistemática, relatos de casos, estudos epidemiológicos e estudos experimentais que abordassem sobre o tema. Os critérios de exclusão foram: cartas ao editor e àqueles que se apresentavam em duplicata.

\section{Estratégias de busca}

As palavras-chaves utilizadas na busca foram selecionadas previamente, identificando-se descritores consultados no MeSH (Medical Subject Headings) e no DeCS (Descritores em Ciências da Saúde). Os descritores foram: "Tendons"; "Tibia"; "Rehabilitation"; "Physiopathology"; "Bloodsupply" e seus correspondentes em português. Após testar os termos isoladamente, identificando grafias alternativas e seus sinônimos, foram estabelecidos os descritores utilizados para compor as estratégias de busca, combinandoos com auxílio de operadores booleanos AND e OR (Tabela 1).

Tabela 1 - Estratégias de busca

\begin{tabular}{cc}
\hline $\mathbf{1}^{\mathbf{a}}$ & Tendons AND (Rehabilitation OR Physiopathology) AND Blood supply \\
\hline $\mathbf{2}^{\mathbf{a}}$ & Tendons AND Tibia AND Blood supply \\
\hline
\end{tabular}

Fonte: Calvalcanti DF, Faleiro TB, 2020.

\section{Seleção dos estudos}

Foi realizada uma leitura seletiva dos títulos e, posteriormente, uma análise dos resumos dos artigos. A pesquisa foi conduzida utilizando o programa State of the Art through Systematic Review (START), disponibilizado pelo Laboratório de Pesquisa em Engenharia de Software (LAPES), da Universidade Federal de São Carlos, São Paulo. Através deste programa, foram listados os artigos e identificados aqueles que se enquadravam nos critérios supracitados, por meio de uma leitura do título e resumo por dois pesquisadores, a fim de incluir apenas trabalhos com 100\% de concordância entre eles. Em caso de dúvida na inclusão do artigo no estudo, nova leitura era feita em conjunto até chegar a uma decisão final. Vale ressaltar que uma busca manual foi feita nas referências dos estudos selecionados.

\section{RESULTADOS E DISCUSSÃO}

\section{Caracterização dos estudos}

Após realização da busca nas bases de dados propostas, obteve-se um total de 552 artigos. Destes, 220 publicações foram excluídas por não atenderem aos critérios de inclusão, por meio da leitura de seus títulos e/ou resumos e 29 por apresentarem duplicidade. Em seguida, excluíram-se mais 23, após a leitura de seus conteúdos na íntegra, por não abordarem a temática, 33 por estarem em outra língua e 226 por não serem disponibilizados na íntegra de forma livre. Esse processo resultou em 21 artigos (Figura 1). 
Figura 1 - Fluxograma dos estudos contemplados na amostra da pesquisa.

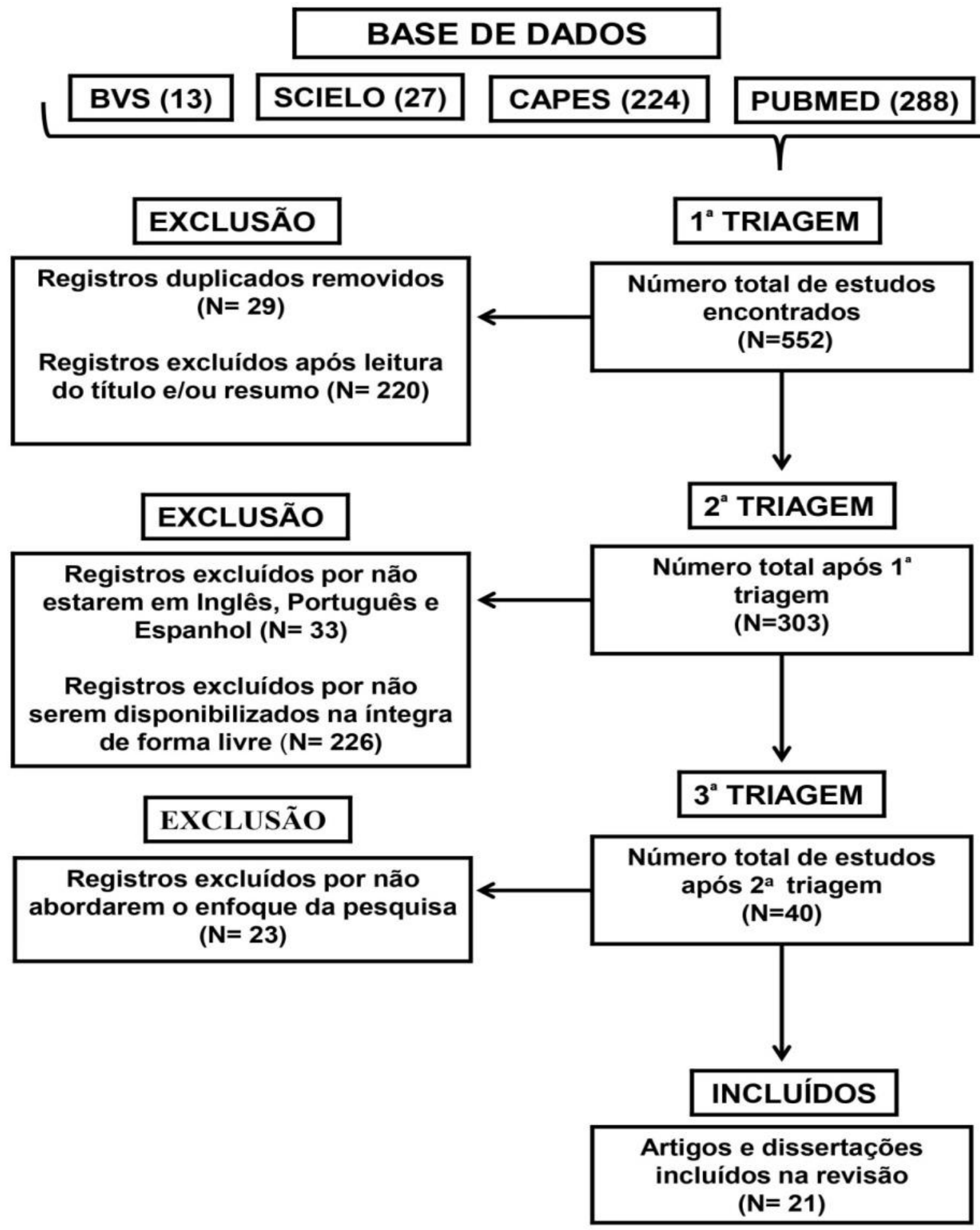

Fonte: Calvalcanti DF, Faleiro TB, 2021

São dispostas as informações extraídas dos trabalhos analisados quanto: ao ano de publicação, língua de publicação, local de origem do estudo e desenhos metodológicos (Tabela 2). Dessa forma, ao verificar as principais características dos 21 estudos elegíveis, que estão organizadas na Tabela 2, observa-se que a maioria desses trabalhos era do tipo pesquisa original $(n=10)$ e dissertações $(n=05)$; publicados principalmente no período de 2016 a $2020(n=10)$, no idioma inglês $(n=13)$ e na região da Europa $(n=09)$ e América do Sul $(\mathrm{n}=06)$. 
Tabela 2 - Características gerais dos estudos incluídos na revisão.

\begin{tabular}{lcc}
\hline Características & $\mathbf{N}=\mathbf{2 1}$ & \% \\
\hline Ano de publicação (N=21) & & \\
\hline $2000-2003$ & 2 & 9,5 \\
$2004-2007$ & 1 & 4,75 \\
$2008-2011$ & 2 & 9,5 \\
$2012-2015$ & 6 & 28,5 \\
$2016-2020$ & 10 & 47,75 \\
\hline Língua (N=21) & & \\
\hline Português & 8 & 38 \\
Inglês & 13 & 62 \\
\hline Local de origem do estudo (N=21) & & \\
\hline América do Norte & 3 & 9,5 \\
Europa & 9 & 52,5 \\
Ásia & 3 & 9,5 \\
América do Sul & 6 & 28,5 \\
\hline Tipo de estudo (N=21) & & \\
\hline Revisão de Literatura & 4 & 19 \\
Revisão Sistemática & 1 & 4,75 \\
Relato de Caso & 1 & 4,75 \\
Pesquisa Original & 10 & 47,75 \\
Dissertação & 5 & 23,75 \\
\hline Total & 21 & 100 \\
\hline
\end{tabular}

Fonte: Calvalcanti DF, Faleiro TB, 2021

\section{Anatomia macroscópica e aspecto histológico}

O Tendão Tibial Posterior (TTP) é o maior e o mais anterior dos tendões mediais do tornozelo bloqueados pelo retináculo atrás do maléolo medial (GUELFI M, et al., 2017). O TTP atua como um estabilizador dinâmico primário de arco longitudinal medial e como inversor principal do mediopé (PRADO MP, 2000; TOHYAMA H, et al., 2009).

A anatomia macroscópica do TTP é bem relatada na literatura: o músculo tibial posterior origina-se da membrana interóssea, tíbia póstero-lateral e fíbula póstero-medial. Desce entre os flexores hallucislongus e flexor digitorumlongus. O músculo da junção tendínea está localizado na região posterior medial na parte do terço distal do bezerro. Na porção intermediária, o TTP flexiona cerca de $80^{\circ}$ rotativamente atrás do maléolo tibial medial(KUBO K, et al., 2019). A inserção distal é colocada no tubérculo do osso navicular, com expansões plantares reforçando a cápsula medial e plantar da articulação talo-navicular (Coxa Pedis) (GUELFI M, et al., 2017).

Na metáfise tibial distal a aponeurose do compartimento posterior profundo divide-se em quatro túneis para acomodar o tibial posterior, o longo flexor comum dos dedos, o longo flexor do hallux e o feixe neurovascular (KUBO K, et al., 2019). O comprimento do tendão varia de 12 a $15 \mathrm{~cm}$ e a seção transversal tem uma forma oval com um diâmetro variando de 06 a 12 mm (GUELFI M, et al., 2017; KUBO K, et al., 2019).

Onde o TTP gira em torno do maléolo medial, é caracterizada por microscopia específica e características macroscópicas: por esse motivo, também é chamado de "Tendão de Gliding" (GUELFI M, et al., 2017; KUBO $\mathrm{K}$, et al., 2019). O planador par é caracterizado pela presença de fibrocartilagem, diferenciando-se por um tendão de estrutura típica de tração. No passado, a literatura considerava a presença da fibrocartilagem como metaplasia ou degeneração, entretanto, muitos estudos mostraram que a fibrocartilagem dentro da parte deslizante do TTP é um componente fisiológico (PRADO MP, 2000; GUELFI M, et al., 2017; KUBO K, et al., 2019).

Erroneamente os tendões eram considerados estruturas avasculares até 1872, quando os estudiosos Ludwig e Schweigger-Seidel demonstraram a existência de vascularização intratendínea. De acordo com a literatura, os tendões recebem o suprimento sanguíneo da junção miotendínea, inserção osteotendínea, na 
região extra-sinovial do paratendão, e região sob a bainha. Entretanto, a literatura elenca que o TTP tem uma zona de suprimento sanguíneo reduzido na região retromaleolar (PRADO MP, 2000).

Ao nível histológico, a vascularização do TTP constitui-se como um sistema simples e uniformizado, no qual compreende uma série de vasos sanguíneos longitudinais com anastomoses transversais e regulares. No entanto, a transição tendão-osso não é considerada fonte de suprimento sanguíneo decisivo para o tendão (TOHYAMA H, et al., 2009).

Os estudos presentes na literatura expõem que a bainha deste tendão mede aproximadamente $07-09 \mathrm{~cm}$ e tem como ponto origem $2,5 \mathrm{~cm}$ proximal à extremidade do maléolo medial, estendendo-se distalmente até a tuberosidade do osso navicular. Pode haver variações do local onde termina a extensão deste tendão, geralmente prolonga-se por 07 centímetros (PRADO MP, 2000; TOHYAMA H, et al., 2009).

O segmento do TTP, localizado internamente a bainha sinovial, recebe o suprimento sanguíneo por meio dos vínculos triangulares e não diretamente da própria bainha, haja vista, que essa porção é desprovida de mesotendão, a estrutura responsável pela origem da vascularização nos tendões (BARROS KD, 2019; KUBO K, 2016). Para Kubo K et al., (2019) as artérias do vínculo proximal são subdivisões originárias dos ramos maleolares mediais provenientes da artéria tibial posterior; enquanto que as da região distal são provenientes do ramo superficial da artéria plantar medial.

\section{Fisiopatologia}

Tem se utilizado varredura radioisótopo e fluxometria Doppler a laser para a investiação mais minuciosa acerca da vascularização dos tendões humanos (DEDERER KM e TENNANT JN, 2019).Com isso, diversos estudos avaliaram a possível correlação entre a mais área do TTP frequentemente afetada por degenerações e a área de hipovascularização neste tendão (PRADO MP, 2000; KUBO K, et al., 2019; TOHYAMA H, et al., 2009; BARROS KD, 2019; DEDERER KM e TENNANT JN, 2019).

A tenossinovite crônica do TTP teve sua primeira descrição atribuída ao estudo de Kulowski (PRADO MP, 2000). Nesse estudo analisa possíveis causas etiológicas e expõem dois casos, um decorrente de um processo infeccioso e o outro de um processo degenerativo. Desde então, a literatura aborda da afecção desse tendão associando-a a traumas, anomalias inflamatórias e infecciosas (PRADO MP, 2000; KUBO K, et al., 2019).

Quanto a degeneração do TTP, observasse na literatura a descrição de uma área crítica onde ocorre uma hipovascularização a 04 centímetros da tuberosidade do navicular, no qual possui maior propensão à processo degenerativos e rupturas no local (KUBO K, et al.,2019; BARROS KD, 2019). A disfunção do tendão tibial posterior (DTTP) é relatada como o principal motivo do pé plano adquirido em pacientes adultos. De antemão, percebe-se que a etiologia ainda é desconhecida, embora seja mais frequente em enfermos do gênero feminino e na sexta década de vida, podendo estar associada a doenças de base como a hipertensão arterial sistêmica, obesidade e diabetes mellitus. Além disso, atribui-se uma região de isquemia do TTP, quando contorna o maléolo tibial, como fator etiológico mais provável (PRADO MP, 2000; KUBO K, et al., 2019; BARROS KD, 2019).

Observa-se como fator predisponente à lesão do TTP a sua trajetória e sua curvatura através do sulco sob o maléolo medial. Apesar das patologias inflamatórias sistêmicas, como a artrite reumatóide, terem uma relação com a insuficiência do tibial posterior como exposto nos estudos de Salomão O et al., (2000) e de Esteves ACF (2016) a controvérsia ainda persiste. O ponto-crítico no estudo deste tendão reside em saber se o pé plano valgo é resultado do processo inflamatório ou da instabilidade decorrente da artropatia subtalar (GUELFI M, et al., 2017).

Está bem relatado na literatura que tendões em estado de normalidade não sofrem rotura quando submetidos ao tracionamento, geralmente resultando em avulsões (PRADO MP, 2000; KUBO K, et al., 2019; TOHYAMA H, et al., 2009; BARROS KD, 2019; SILVA DJF, 2012; GEREMIA JM, 2011; SANHUDO JAV, 2012; GUELFI M, et al., 2017). Para que ocorra a ruptura na trajetória tendínea necessita de uma atenuação parenquimatosa do mesmo (PRADO MP, 2000; GUELFI M, et al., 2017). 
O interesse em correlacionar os déficits vasculares com o sítio mais frequente dessa afecção é uma preocupação desde a década de 50 (PRADO MP, 2000). Recentemente a literatura parece ter entrado em consenso quanto à região constantemente degenerada, localizada a $04 \mathrm{~cm}$ da sua inserção na tuberosidade do osso navicular, possa coincidir com a área de hipovascularização (KUBO K, et al., 2019; TOHYAMA H, et al., 2009; BARROS KD, 2019).

Apesar do estudo da rede vascular possuir diversos métodos para sua avaliação, nenhum obteve a excelência diagnóstica (PRADO MP, 2000; TOHYAMA H, et al., 2009). Comumente, as técnicas mais aplicadas são as injeções intra-arteriais para coloração ou contraste radiológico, o que permite mapeamento dessa rede em questão; e a visão direta por meio de ressecção histológica. A correlação direta da hipovascularização com o sítio mais afetado pela da rotura é discutível, assim como, o seu papel determinante único (PRADO MP, 2000; GUELFI M, et al., 2017; BARROS KD, 2019).

Nesse aspecto, a existência de uma luxação do TTP congruente a uma fratura ou a um trauma grave do tornozelo já é bastante reconhecida pela literatura científica, enquanto que apenas a sua luxação isolada é extremamente rara seu acontecimento como expõe por seus pouquissimos casos (DEDERER KM e TENNANT JN, 2019; SALOMÃO O, et al., 2000).

Dentre as lesões traumáticas desse tendão em especifico, observa-se grande ênfase voltada para o diagnóstico e a correta terapêutica da tenossinovite e da ruptura degenerativa do TTP; quanto aos casos de luxação e subluxação ao nível do tornozelo, nota-se que ocorre de forma muito mais frequente nos fibulares (KUBO K, 2016; SALOMÃO O, et al., 2000).

Atualmente quanto às linhas de pesquisa acerca da etiopatogenia, nota-se uma centralização a respeito das modificações do tecido colágeno observadas na população de faixa etária mais avançada e o déficit da vascularização do TTP (PRADO MP, 2000; DEDERER KM e TENNANT JN, 2019; SALOMÃO O, et al., 2000; ESTEVES ACF, 2016). A trama vascular intra e extratendínea estão bem descritas na literatura; entretanto, permanece questionável a vascularização específica de determinados tendões.

\section{Manejo terapêutico}

As lesões tendíneas do uso excessivo são muito comuns sintomas para atletas recreativos e competitivos (KUBO K, et al., 2019). Possui a etiologia incerta e controversa, a literatura inclui a associação com patologias infecciosas e reumáticas, traumas anteriores, anatomia inerente do local, função e hipovascularização (PRADO MP, 2000).

Considera-se que a cicatrização de lesões por estes tratamentos contribui para a melhoria do sangue circulação dos tecidos lesionados (KUBO K, et al., 2019; TOHYAMA H, et al., 2009; WEZENBEEK E, et al., 2018). De fato, estudos anteriores usando laser vermelho mostraram que o volume sanguíneo saturação de oxigênio (StO2) dos tendões aumentou significativamente por aquecimento, acupuntura e terapia hiperbárica (KUBO K, et al., 2019; TOHYAMA H, et al., 2009; KUBO K, 2016; WEZENBEEK E, et al., 2018).

Sabe-se que a acupressão, compressão pneumática intermitente e mecânica vibração trazer efeitos terapêuticos para aliviar o desconforto e dor do corpo. Estudos anteriores também mostraram que 0 comportamento simpático atividades parassimpáticas alteradas pela acupuntura e acupressão (KUBO K, et al., 2019; REES JD, et al., 2014; WEZENBEEK E, et al., 2018). Considerando esses pontos, é possível que a compressão mecânica no tendão possa causar alterações na circulação sanguínea dos não tratados (contralateral) tendão, bem como o tendão tratado, na mesma maneira como a acupuntura (KUBO K, et al., 2019; KUBO K, 2016).

As principais conclusões do estudo de Kubo $\mathrm{K}$ et al.,(2019) foram as seguintes: (1) a alterações induzidas pela acupuntura na circulação sanguínea do tendão (aumentos repentinos de $\mathrm{THb}$ e StO2 do tendão tratado durante a acupuntura com manipulação para cima e para baixo e aumentos graduais de $\mathrm{THb}$ e $\mathrm{StO} 2$ dos pacientes tratados e não tratados tendões após a remoção da agulha) concordou com estudos anteriores; e (2) as alterações na circulação sanguínea tendão por compressão intermitente foram semelhantes àquelas induzidas pela acupuntura. 
Infelizmente, os mecanismos que resultam no aumento da $\mathrm{O} T H \mathrm{~b}$ do tendão após compressão intermitente é desconhecido (KUBO K, et al., 2019; KUBO K, 2016). Pesquisadores anteriores afirmaram que a redução da pressão causada pela compressão intermitente levou a um aumento gradiente de perfusão arteriovenosa, permitindo aceleração fluxo sanguíneo (KUBO K, et al., 2019; TOHYAMA H, et al., 2009; KUBO K, 2016). Além disso, o maior fluxo velocidade aumenta a tensão de cisalhamento nas paredes endoteliais dos vasos, o que aumentou a produção de óxido nítrico. Portanto, o aumento da $\mathrm{THb}$ do tendão tratado com a compressão intermitente pode ser esclarecido pelos mecanismos descritos acima (KUBO K, et al., 2019; TOHYAMA H, et al., 2009; KUBO K, 2016; BITTAR CK, et al., 2018; PINGEL J, et al., 2013).

A ruptura parcial ou completa de um tendão tem como causa principal a sobrecarga excêntrica da unidade musculotendínea, porém, tanto fatores intrínsecos como alterações degenerativas das fibras de colágeno, vascularização, necrose dos tenócitos e a influência genética, quanto fatores extrínsecos como condições de superfície desfavorável e a utilização de calçados inadequados podem estar associados a essa ruptura (PRADO MP, 2000; KNOBLOCH K, 2007; BITTAR CK, et al., 2018; PINGEL J, et al., 2013).

O diagnóstico é confirmado pela história clínica e pelo exame físico do paciente, caracterizados por dor e fraqueza no alongamento do membro, limitação funcional e alteração na palpação do tendão (BITTAR CK, et al., 2018; REES JD, et al., 2014). A ressonância magnética tem sido usada de maneira complementar para auxiliar no estadiamento, diagnóstico das lesões, planejamento cirúrgico e nos diagnósticos diferenciais (BITTAR CK, et al., 2018; DE JONGE S, et al., 2013). Haja vista, que este exame imaginológico fornece informações mais abrangentes sobre a morfologia interna do tendão e das estruturas adjacentes, sendo útil na avaliação dos vários estágios de degeneração crônica e na diferenciação entre lesões traumáticas neste tendão (BARROS KD, 2019).

Considera-se a idade do paciente sendo um fator decisivo na escolha da terapêutica aplicado, avaliando entre tratamento fisioterápico ou cirúrgico (BITTAR CK, et al., 2018; DE JONGE S, et al., 2013). Nesse aspecto, a abordagem cirúrgica se faz necessária ao visar o restabelecimento da capacidade funcional. Entretanto, dependendo da dimensão da instabilidade, da presença de deformidades e do quadro clínico do paciente justifica realizar a artrodese (subtalar ou tríplice), no qual se classifica como cirurgia estabilizadora. Em casos de estadiamento mais avançado (estágios III e IV), o tratamento mais indicado é a artrodese (BITTAR CK, et al., 2018; PINGEL J, et al., 2013; DE JONGE S, et al., 2013).

O reparo cirúrgico das tendinopatias mostrou-se extremamente efetivo na reabilitação funcional dos pacientes, com uma baixa taxa de complicações e elevado grau de satisfação dos pacientes (BITTAR CK, et al., 2018; PINGEL J, et al., 2013; DE JONGE S, et al., 2013; MAGNAN B, et al., 2014).

\section{CONSIDERAÇÕES FINAIS}

A disfunção e a degeneração do TTP é uma condição comum, mas muitas vezes mal diagnosticada. Causa uma deformidade progressiva e dolorosa noemada como "pé chato". A anatomia e vascularização expõem a região do TTP a distúrbios degenerativos importantes ao ponto até de uma ruptura. Nas últimas décadas, dados da literatura mostrou uma zona de suprimento sanguíneo baixa do tendão de Gliding, que parece estar diretamente associado ao local de maior prevalência das lesões degenerativas. Entretanto, os mecanismos vasculares que resultam na degeneração deste tendão ainda são desconhecidos e se faz necesário estudos mais aprofundados nesse aspecto.

\section{REFERÊNCIAS}

1. BARROS KD. Tratamento da rotura crônica do tendão de Aquiles. Dissertação (Mestrado Integrado em Medicina) Universidade do Porto, Porto, 2019; 42 p.

2. BITTAR CK, et al. Tendinopatias do pé e tornozelo. Rev Cienc Med, 2018;27(2):59-64.

3. DEDERER KM, TENNANT JN. Anatomical and functional considerations in Achilles tendon lesions. Foot Ankle Clin N Am, 2019;24:371-85.

4. DE JONGE S, et al. Relationship between neovascularization and clinical severity in Achilles tendinopathy in 556 paired measurements. Scand J Med Sci Sports, 2013:1-6. 
5. ESTEVES ACF. Avaliação do biopolímero extraído da cana-de-açúcar no modelo experimental de lesão do tendão do calcâneo de ratos. Tese (Doutorado em Biotecnologia e Saúde) - UFP, Recife, 2016; 115 p.

6. GEREMIA JM. Ruptura total do tendão de Aquiles: propriedades mecânicas tendíneas em indivíduos submetidos a diferentes protocolos de reabilitação. Dissertação (Mestrado em Educação Física) - UFRGS, Porto Alegre, $2011 ; 122$ p.

7. GUELFI M, et al. Anatomy, pathophysiology and classification of posterior tibial tendon dysfunction. Eur Rev Med Pharmacol Sci, 2017;21:13-9.

8. ISHIGAKI T, KUBO K. Effects of eccentric training with different training frequencies on blood circulation, collagen fiber orientation, and mechanical properties of human Achilles tendons in vivo. EJAP, 2018:1-10.

9. KNOBLOCH K. Eccentric training in Achilles tendinopathy: is it harmful to tendon microcirculation. Br J Sports Med, 2007;41:1-5.

10. KUBO K, et al. Acupuncture and intermittent compression induced changes in blood circulation of tendon. JACM, 2019;1-8.

11. KUBO K. Metabolic Tendon Disorders-Diagnosis and Management. Blood supply, 2016:27-33.

12. MAGNAN B, et al. The pathogenesis of Achilles tendinopathy: a systematic review. Foot and Ankle Surgery, 2014;20:154-59.

13. PINGEL J, et al. The microvascular volume of the Achilles tendon is increased in patients with tendinopathy at rest and after a 1-hour treadmill run. Am J Sports Med, 2013;41:2400-08.

14. PRADO MP, et al. Estudo da densidade vascular do tendão do músculo tibial posterior em cadáveres. Rev Bras Ortop, 2000;35(3):53-66.

15. REES JD, et al. Tendons - time to revisit inflammation. Br J Sports Med, 2014; 48: 1553-1557.

16. SALOMÃO $O$, et al. Luxação traumatic do tendão tibial posterior: Relato de caso e revisão da literatura. Rev Bras Ortop, 2000;29(7):471-74.

17. SANHUDO JAV. A relação da discrepância no comprimento dos membros inferiores com a disfunção do tendão tibial posterior. Dissertação (Doutorado em Medicina) - UFRGS, Porto Alegre, 2012; 82 p.

18. SILVA DJF. Rotura do Tendão de Aquiles. Dissertação (Mestrado Interado em Medicina) - FMUP, Porto, $2012 ; 44$ p.

19. TOHYAMA H, et al. Revascularization in the tendon graft following anterior cruciate ligament reconstruction of the Knee: Its mechanisms and regulation. Chan Gung Med J, 2009;32(2):133-39.

20. WEZENBEEK E, et al. Activity-induced increase in Achilles tendon blood flow is age and sex dependent. Amer $\mathrm{J}$ Sports Med, 2018:1-9.

21. WEZENBEEK E, et al. The role of the vascular and structural response to activity in the development of Achilles tendinopathy. Amer J Sports Med, 2018:1-8. 